\title{
Clinical practice guidelines appraisal and utilisation by Pharm.D. students
}

\author{
Dixon Thomas $^{1}$ (D), Seeba Zachariah ${ }^{1}$ (D) , Julie Akers² ${ }^{\text {(D) }}$ \\ ${ }^{1}$ Gulf Medical University College of Pharmacy, United Arab Emirates \\ ${ }^{2}$ Washington State University College of Pharmacy and Pharmaceutical Sciences, USA
}

\author{
Keywords \\ Clinical Practice Guidelines \\ Pharmacy Students \\ Learning Outcomes \\ Bloom's Cognitive Levels \\ Professional Identity \\ Correspondence \\ Dr Dixon Thomas \\ Gulf Medical University \\ College of Pharmacy \\ Jurf \\ Ajman \\ United Arab Emirates PO Box 4184 \\ dixon.thomas@gmail.com
}

\begin{abstract}
Objective: To assess the appraisal and utilisation of clinical practice guidelines (CPGs) by pharmacy final-year students. Methods: Four CPGs of 2017 were appraised by pharmacy students using the AGREE-II instrument. The utilisation of CPGs by pharmacy students was assessed using a cross-sectional survey and results were analysed using the Wilcoxon Signed Ranks test. Results: Of the six AGREE-II domains for CPGs, two domains were mid to high (>90\%), two domains $76 \%$ each, and the last two domains $<60 \%$. The utilisation of CPGs by pharmacy students as a reference in reports/presentations were high. Students felt that they are relatively better able in lower Bloom's cognitive levels (knowledge and comprehension) than the higher abilities of application, evaluation, and creation of clinical guidelines, ( $p$-value 0.006). Conclusions: Among AGREE-II domains, lower scores for CPGs were in editorial independence and applicability. Evaluation of CPGs by students is a useful tool to comprehend differences in their quality. More CPGs-related training in higher Bloom's cognitive levels is required for the Pharm.D. students.
\end{abstract}

\section{Introduction}

The Institute of Medicine (now known as National Academy of Medicine) defined clinical practice guidelines (CPGs) as 'statements that include recommendations intended to optimise patient care that is informed by a systematic review of evidence and an assessment of the benefits and harms of alternative care options' (Institute of Medicine, 2011: p.4). Doctor of Pharmacy (Pharm.D.) students undergo several months of Advanced Pharmacy Practice Experience (APPE) towards the end of their programme. In clinical rotations, the students need to use CPGs extensively to support their clinical learning. In clinical settings, students use a deep learning approach, allowing for initial base knowledge, followed by increased understanding and application (Emilia et al., 2012).

Evidence-based practice develops through identification, critical appraisal and application of best quality evidence (Thomas et al., 2019). Learning outcomes related to CPG are part of pharmacy education. Bloom's taxonomy of cognitive levels is knowledge, comprehension, application, evaluation and creation. Knowledge and comprehension are considered at low levels and the rest are considered at higher levels. Higher cognitive levels like application, evaluation and creation are especially used in clinical learning involving direct patient care (Austin, 2016). CPGs are commonly used in clinical practice and learning. Learning using CPG prepares students to follow the standards of care. The guidelines should be critically appraised before applying it to practice and implementing their use into skills training with students (Brown, 2015).

Choosing proper guidelines to utilise in practice is part of developing a professional identity. Specific training programmes have the potential to improve professional identity among pharmacy students. Increased professional identity results in more autonomy and intrinsic motivation (Mylrea et al., 2019). 


\section{Methods}

\section{Appraisal of CPG}

The Appraisal of Guidelines for REsearch and Evaluation (AGREE) Instrument evaluates the process of clinical guideline development and the quality of reporting. The AGREE-II is both valid and reliable and comprises 23 items organised into the original six quality domains (Brouwers et al., 2010). Three Ph.D. students appraised four mainstream CPG (two cardiovascular and two kidney guidelines, all published in 2017) to observe patterns of quality among six domains of the AGREE-II instrument. The students under supervision performed the appraisal and reached upon consensus scores.

\section{Utilisation of CPG}

A cross-sectional survey was conducted among Pharm.D. APPE students after six months of their final-year of experiential education, approximately one month before graduation. This was a population-based survey using Google Forms and the content of the survey form is included (Appendix A). Out of 29 interns, 26 completed the survey, a $90 \%$ response rate. The students were asked to rate their abilities against five low- and five high-level Bloom's cognitive competencies regarding clinical guidelines using Likert's scale 'Strongly Agree' to 'Strongly Disagree' scoring 4 to 0 . Wilcoxon-Signed Ranks Test was used to find if there was a significant difference between the sum of low- and high Bloom's cognitive level scores at $p$-value 0.05 .

This research was conducted among final-year Pharm.D. students at the Gulf Medical University, UAE. The University has an academic health system with hospitals, clinics, and pharmacies. The students had experiences in direct patient care performing clinical pharmacy services under supervision. Towards the end of the experiential year, a group of preceptors conducted this study using an online survey for their students who were about to graduate. The Institutional Review Board approved this research. Consent was taken from the students while collecting data.

\section{Results}

\section{Appraisal of CPG}

Though there is no clear threshold for an acceptable quality of guidelines, generally, a score above $70 \%$ is considered good quality as described by the AGREE manual (Brouwers et al., 2010). In this appraisal the Domain 1: Scope and Purpose scored 94\% (SD \pm 7 ) and Domain 4: Clarity of Presentation scored 99\% (SD \pm 3 ). Domain 2: Stakeholder Involvement 76\% (SD \pm 29$)$ and Domain 3: Rigour of Development 76\% (SD \pm 20$)$; were reasonably good quality with considerable variation among guidelines. Domain 5: Applicability 57\% (SD \pm 11$)$ and Domain 6: Editorial Independence 36\% (SD \pm 43 ) were scored relatively lower.

\section{Utilisation of CPGs}

Based on student responses on the survey for the lower Bloom's cognitive levels were as follows: $92 \%$ of them have a habit of reading relevant guidelines if available; $92 \%$ of them are able to recall key points of common CPGs relevant to their practice area; $92 \%$ could appreciate that CPGs are generally evidence-based; $92 \%$ could appreciate that CPGs usually are patient-centred; and $85 \%$ of them could define their practice as a standard of care by use of guidelines.

The same student group responses regarding higher Bloom's cognitive levels were $81 \%$ of them could retrieve appropriate guidelines from internal or external resources in a reasonable time; $85 \%$ could appraise guidelines for methodological rigour as there are controversial or biased guidelines; $81 \%$ could incorporate evidence in guidelines with local experience and patient preferences; $73 \%$ could individualise recommendations in a guidelines to specific patient needs; and $77 \%$ of them could propose revision of internal hospital guidelines or protocols.

A statistically significant difference exists between the agreement of students on their lower and higher Bloom's cognitive levels ( $p$-value 0.006).

The Pharm.D. interns used many popular CPGs in their learning ranging from 2 (7\%) for GOLD guidelines to 28 (96\%) for ACC/AHA guidelines. Twenty-five (86\%) used the American Society of Health-System Pharmacy (ASHP) guidelines. Only ten (34\%) used the Institute for Safe Medication Practices (ISMP) guidelines. More than 20 (70\%) used Google to access guidelines or accessed it through information provided in UpToDate, Clinicalkey, or even textbooks. Guideline usage was common for student presentations (>80\%) and answering drug information queries (>70\%). Only nine (31\%) felt guidelines were readily available, and only seven (24\%) thought guidelines have more applicable information than clinical trials. Seventeen (59\%) interns rated their abilities to appraise guidelines as average-level. 


\section{Discussion}

Appraising a group of popular mainstream CPGs gave an insight into the challenges faced by time-tested guidelines. Though the rigour of development and clarity of presentation were well-taken care, it was difficult to communicate applicability. It was a valuable learning experience for the three students who participated in the appraisal of the guidelines. As information was insufficient and just one item in Domain 6: Editorial Independence, the authors could not derive a conclusion on it. Capacity building and collaborative efforts are necessary to increase the application of best quality evidence from appropriate guidelines (Novo et al., 2016).

Higher Bloom's cognitive levels regarding CPGs need improvement with more effective learning strategies. Required knowledge gains related to CPGs are necessary to effectively move into deeper levels of learning during APPE rotations. Opportunities for students to learn at a higher level earlier in the curriculum will improve student performance in APPE (Gregory et al., 2019). To improve pharmacists' professional identity, it is important to guide interns to use relevant CPGs characteristic to the profession. Although the use of CPGs is recognised as a way to increase professional identity, a pharmacist might lack professional identity due to many reasons (Donohoe et al., 2016).

\section{Conclusions}

Assessment and utilisation of CPGs are useful tools for pharmacy students to improve professional identity and deeper learning. The use of CPGs early in the curriculum shall provide enough time to improve learning in higher Bloom's cognitive levels.

\section{Acknowledgment}

The authors thank Prof. Jayadevan Sreedharan of Gulf Medical University for statistical support.

\section{References}

Austin, Z. (2016). How to design and use learning objectives in clinical teaching. The Pharmaceutical Journal, 26 (296), 7885

Brouwers, M.C., Kho, M.E., Browman, G.P., Burgers, J.S., Cluzeau, F., Feder, G., Fervers, B., Graham, I.D., Grimshaw, J., Hanna, S.E., Littlejohns, P., Makarski, J., Zitzelsberger, L., \& AGREE Next Steps Consortium (2010). AGREE II: advancing guideline development, reporting and evaluation in health care. CMAJ: Canadian Medical Association Journal/Journal de I'Association Medicale Canadienne, 182(18), E839-E842. https://doi.org/10.1503/cmaj.090449
Brown D.L. (2015). Rethinking the Role of Clinical Practice Guidelines in Pharmacy Education. American Journal of Pharmaceutical Education, 79(10), 148. https://doi.org/10.5688/ ajpe7910148

Donohoe, K.L., Drisaldi, A.G., Alotaibi, F.M., Bonas, T.N., Shibley, E.M., \& Slattum, P.W. (2016). Student Knowledge, Skills, and Self-Efficacy Gains After Completing an Advanced Pharmacy Practice Experience in Geriatrics. American Journal of Pharmaceutical Education, 80(9), 156. https://doi.org/10.5688/ ajpe809156

Emilia, O., Bloomfield, L., \& Rotem, A. (2012). Measuring students' approaches to learning in different clinical rotations. BMC Medical Education, 12, 114. https://doi.org/10.1186/1472-6920-12-114

Gregory, P., \& Austin, Z. (2019). Pharmacists' lack of profession-hood: Professional identity formation and its implications for practice. Canadian Pharmacists Journal: CPJ/Revue des Pharmaciens du Canada: RPC, 152(4), 251-256. https://doi.org/10.1177/1715163519846534

Institute of Medicine (2011). Committee on Standards for Developing Trustworthy Clinical Practice Guidelines. (2011). In Clinical Practice Guidelines We Can Trust, (Eds. R. Graham, M. Mancher, D.M. Wolman, S. Greenfield, E. Steinberg). Washington (DC), National Academies Press, US. https://doi.org/10.17226/13058

Mylrea, M.F., Gupta, T.S., \& Glass, B.D. (2019). Design and Evaluation of a Professional Identity Development Program for Pharmacy Students. American Journal of Pharmaceutical Education, 83(6), 6842. https://doi.org/10.5688/ajpe6842

Novo, A., Subotic-Popovic, A., Strbac, S., Kandic, A., \& Horga, M. (2016). Application of Agree II Instrument for Appraisal of Postpartum Hemorrhage Clinical Practice Guidelines in Bosnia and Herzegovina. Acta informatica medica: AIM: journal of the Society for Medical Informatics of Bosnia \& Herzegovina: Casopis Drustva za medicinsku informatiku BiH, 24(3), 211-214. https://doi.org/ 10.5455/aim.2016.24.211-214

Thomas, D., de Sousa, I. C., Woods, D.J., Herman, R.A., \& Baker, D.E. (2019). Drug Information Training for Pharmacists. In Clinical Pharmacy Education, Practice and Research, (Ed. D. Thomas), Amsterda, Elsevier. pp. 191-199. https://doi.org/10.1016/B978-012-814276-9.00013-1

\section{Appendix A}

\section{Data Collection form}

1. Tick ALL the following Clinical Practice guidelines you read (even partly) at least once in your APPE for a case presentation or solving a case question?

- ACC/AHA Guidelines

- Eighth Joint National Committee (JNC 8) Guidelines

- Infectious Diseases Society of America (IDSA) Guidelines

- American Society for Health-System Pharmacists (ASHP) Guidelines

- Global Initiative for Chronic Obstructive Lung Disease (GOLD) Guidelines

- American College of Clinical Pharmacy (ACCP) Guidelines

- Institute of Safe Medication Practices (ISMP) Guidelines

- National Institute for Health and Care Excellence (NICE) Guidelines

- American College of Physicians (ACP) Guidelines

- American Academy of Family Physician (AAFP) Guidelines

- International Society for Pharmacoeconomics \& Outcomes Research (ISPOR) Guidelines 
- Thumbay Hospital Internal Guidelines or Protocols

- Ministry of Health, UAE Guidelines

- Other:

2. How do you access information (full or partial) in guidelines? Tick all that apply

- Through Medscape

- Through UpToDate

- Through ClinicalKey

- From Textbooks like Dipiro Pharmacotherapy

- From Google Search

- Directly from Guidelines websites like the NICE website or AHRQ website, or ISMP website

- Guidelines as published articles in a journal

- Emailed by preceptors or faculty

- Emailed by your classmate

- Others

3. Tick all aspects help or motivate you to use clinical guidelines Preceptors and Doctors appreciate the use of guidelines

- Easy availability of guidelines

- Case Presentations need to mention guidelines

- Guidelines presentation/discussion as part of a rotation

- Drug Information answer need guidelines referred

- More applicable than Clinical Trial information

- Reputed guidelines provide trusted information

- Others

4. When you wanted to read clinical guidelines, you got the full version of it as it is very much available

- Strongly Agree, Agree, Neutral, Disagree, Strongly Disagree

5. Select your current skill level in using Clinical Guidelines?

- I only read and use it in my case presentations

- In addition to the above, I use them in my discussions and the interventions or patient-specific queries answered

- In addition to the above, I check the strength of evidence and class of recommendations to recognize some recommendations as strong and some as week as mentioned in the guidelines. Presented/discussed my finding in internal presentations.

- In addition to the above, I am aware of the AGREE format for guidelines and GRADE level of evidence. I evaluate or appraise guidelines and presented my findings in an event outside my usual APPE presentations

- In addition to the above, I did communicate my concern of a particular guideline to those who made it, or I published a writeup about issues of guidelines because my abilities in evaluating guidelines are high

6. How do you think while graduation you are able to perform the following provided the conditions are ideal, consider it just depends on your ability alone;

(Lower Bloom's cognitive level)*

- Show a habit of reading relevant guidelines if available

- Recall key points of common clinical guidelines relevant to their practice area

- Appreciate that clinical guidelines are generally evidence-based

- Appreciate that clinical guidelines are generally patient-centred

- Define practice as a standard of care by use of guidelines
(Higher Bloom's cognitive level)*

- Retrieve appropriate guidelines from internal or external resources in a reasonable time

- Appraise guidelines for methodological rigour as there are controversial or biased guidelines

- Incorporate evidence in guidelines with local experience and patient preferences

- Individualise recommendations in a guideline specific to our patient needs

- Propose revision of internal hospital guidelines or protocols

*(Note: When the survey was rolled out, the ten abilities mentioned above were mixed and it was not mentioning the lower or higher level of Bloom's Cognitive taxonomy.)

7. Provide any additional comments about the use of Clinical Guidelines on APPE learning and practice 